\title{
Un an déjà !
}

\section{One Year Already!}

\section{P. Poisbeau (Éditeur de section, vice-président de la SFETD)}

(C) Lavoisier SAS 2019

La revue Douleur et Analgésie est le journal officiel de la Société française d'étude et de traitement de la douleur (SFETD) depuis près d'un an. Avec son comité de rédaction renouvelé, une nouvelle dynamique s'est installée, et de nouvelles rubriques ont vu le jour afin de présenter le plus fidèlement possible l'activité de recherche et les pratiques cliniques de la communauté francophone travaillant dans le domaine. En permettant à chaque professionnel, quel(le) que soit sa spécialité ou son domaine de compétence, de présenter ses travaux. Nous sommes également particulièrement heureux d'être accompagnés par nos amis suisses, belges et canadiens dans cette aventure scientifique.

Ce numéro est un reflet de cette ligne éditoriale inspirée par l'activité de la SFETD, que j'ai tenté de vous rappeler très brièvement. Ce numéro est fidèle à notre ligne éditoriale.

\section{Douleur animale}

Cette question a fait l'objet de nombreuses réglementations depuis les 20 dernières années, au niveau européen et français, transformant complètement les pratiques dans les laboratoires de recherche. Les actualités liées à la qualité de la production animale font surgir de nouveaux questionnements de société. Dans ce numéro, deux articles sont proposés par Bernard Calvino et Thierry Poitte. Le premier article, proposé par Bernard Calvino, pose la question de la douleur chez certains poissons. Comment ces travaux font-ils écho aux explorations pratiquées chez d'autres espèces et en particulier les Mammifères ? La douleur des Mammifères, comme le chien de compagnie, est également une préoccupation en clinique vétérinaire. Thierry Poitte, initiateur et animateur du réseau CAP Douleur, s'intéressant particulièrement à la douleur du chien de compagnie, fait le point sur les pratiques françaises, l'élaboration de recommandations, le partage d'expérience et le développement d'outils innovants utilisant éventuellement l'intelligence artificielle.

\section{Recommandations}

Les recommandations sont essentielles pour nos pratiques professionnelles. Quatre auteurs éminents, dont Patrice Queneau (de l'Académie nationale de médecine), se sont associés pour présenter dans ce numéro des recommandations pour la meilleure prise en charge des douleurs chroniques. Issu d'un groupe de travail pluriprofessionnel soutenu par la SFETD, l'article intitulé « Les recommandations de Lille » présente les recommandations diagnostiques pour la prise en charge thérapeutique des syndromes douloureux régionaux complexes. Un travail remarquable du groupe et de ses animateurs.

\section{Des mots, des maux}

Pour ce numéro, trois articles originaux sont proposés avec des thématiques variées. Cette rubrique évoque ainsi l'intérêt de travailler sur la mobilité psychique des patients parmi les approches alternatives aux effets délétères de la douleur chronique (Lionet \& Bioy) et les difficultés de la communication médecin-malade dans les annonces de mort, l'accompagnement d'un impossible à penser et de l'impact de l'absence d'incertitude pour l'accompagnement des patients (Alric). Le dernier article présente un outil d'évaluation du statut psychodynamique des patients douloureux chroniques visant à donner des indications en vue d'une proposition de diagnostic et de traitement (Minjard et Barfety-Servignat).

P. Poisbeau $(\bowtie)$

Université de Strasbourg

Centre National de la Recherche Scientifique (CNRS)

e-mail : poisbeau@inci-cnrs.unistra.fr 